\title{
Mortalidad posoperatoria después de un año del evento quirúrgico en una cohorte de pa- cientes adultos mayores
}

\author{
One-year postoperative mortality in a cohort \\ of older patients
}

Pascuala Ebner ${ }^{1, a}$, Afaf Rumie ${ }^{1, a}$, Francisca González ${ }^{1, a}$, Rodrigo Gutiérrez ${ }^{2, b, c}$, Antonello Penna $2, b, c$

\begin{abstract}
Aim: To determine one-year postoperative mortality in patients older than 65 years at the Hospital Clínico de la Universidad de Chile. Material and Methods: After approval by the ethics committee, a random sample of 235 patients was obtained from 2,832 patients $\geq 65$ years who underwent surgery that required general or regional anesthesia. This sample size was calculated to detect a mortality incidence of $10 \% \pm 5 \%$, with a power of $80 \%$, an $\alpha$ error of 0.05 , and a loss of $10 \%$. We recorded the demographic variables together with the Charlson Comorbidity Index (CCI) score from the electronic medical records. While the date of mortality was obtained from the Civil Registry. Results: We studied 233 patients with an age of $73.1 \pm 6.3$ years, $52.4 \%$ were women, and a mean $\mathrm{CCl}$ score of 4 (2-11) points. In total $65.7 \%$ underwent general anesthesia, 34.3\% underwent regional anesthesia, and $24 \%$ underwent major surgery. Mortality at 30 days was $1.3 \%$ and at one year it was $6 \%$. The patients who died were older and had a higher $\mathrm{CCl}$, especially due to a higher incidence of dementia and solid tumors with metastases. Conclusions: In our study, postoperative mortality at one year was lower than those reported in older adult patients, and deceased patients were older with more comorbidities, especially with solid tumors with metastases and dementia.
\end{abstract}

\section{Key words:}

Older adults,

postoperative period, mortality,

Chile

Estudiante de Medicina.

Anestesiólogo, MD-PhD.

Hospital Clínico de la Universidad de Chile, Escuela de Medicina de la Universidad de Chile.

Departamento de Anestesiología y Medicina Perioperatoria, Facultad de Medicina y Hospital Clínico de la Universidad de Chile.

Centro de Investigación Clínica Avanzada (CICA), Facultad de Medicina y Hospital Clínico de la Universidad de Chile.

Fecha de recepcion: 07 de octubre de 2020

Fecha de aceptación: 06 de enero de 2021

\section{ORCID}

0000-0002-8626-4415

Fuente de Financiamiento: sin fuentes de financiamiento.

Conflicto de interés: sin conflictos a declarar.

Correspondencia:

Antonello Penna

apenna@uchile.cl 


\section{RESUMEN}

Objetivo: Determinar la mortalidad al primer año postoperatorio de pacientes mayores de 65 años en el Hospital Clínico de la Universidad de Chile. Materiales y Métodos: Tras la aprobación del comité de ética, se obtuvo una muestra aleatoria de 235 pacientes de un total de 2.832 pacientes $\geq 65$ años sometidos a una cirugía que requirió de anestesia general o regional. Este tamaño muestral fue calculado para detectar una incidencia de mortalidad de $10 \% \pm 4 \%$, con un poder de $80 \%$, un error $\alpha$ de 0,05 y una pérdida de $10 \%$. Se registraron los antecedentes demográficos, se calculó el puntaje de Charlson Comorbidity Index (CCI) y se consignó la mortalidad desde el Registro Civil. Resultados: Se estudiaron 233 pacientes con una edad de 73,1 $\pm 6,3$ años, un $52,4 \%$ fueron mujeres y una mediana del puntaje $\mathrm{CCl}$ de 4 (2-11) puntos. En total $65,7 \%$ fue intervenido bajo anestesia general y $34,3 \%$ bajo anestesia regional, y $24 \%$ fue sometido a una cirugía de alta complejidad. La mortalidad a los 30 días fue de 1,3\% y al año fue de $6 \%$. El grupo de pacientes fallecidos se caracterizó por ser más añoso y tener un $\mathrm{CCl}$ mayor, especialmente por una mayor incidencia de demencia y tumores con metástasis. Conclusiones: En nuestro estudio se observó una mortalidad postoperatoria al año menor a lo reportado internacionalmente en pacientes adultos mayores. La mortalidad fue mayor en pacientes de mayor edad, con un mayor número de comorbilidades, con tumores sólidos con metástasis y demencia.

\section{Palabras clave:}

Adulto mayor, período posoperatorio, mortalidad,

Chile.

\section{Introducción}

L as nuevas técnicas quirúrgicas y anestésicas han permitido disminuir de manera significativa la -mortalidad intraoperatoria de cirugías no cardiacas, a pesar de la mayor edad y comorbilidad de los pacientes que se someten a ellas[1]. Sin embargo, la mortalidad posoperatoria a mediano y largo plazo no ha disminuido, más bien se ha mantenido estable entre el $1 \%$ y $3 \%$ a los 30 días tras el evento quirúrgico en mayores de 40 años[1]-[6], mientras que la mortalidad global en el primer año tras una cirugía se ha reportado de 5,5\%, aumentando a 10,3\% en pacientes mayores de 65 años[4]. Las causas de muerte en el período postoperatorio, tanto a mediano como a largo plazo, se deben principalmente a complicaciones quirúrgicas, al avance de su condición preexistente, por ejemplo, el avance de la patología oncológica, y a eventos cardiovasculares, como infarto agudo al miocardio (IAM)[1],[4]. Registrar y reportar las cifras de mortalidad postoperatoria de una institución, de la región y del país, es fundamental para guiar las conductas clínicas con los pacientes, con lo que se podría optimizar de uso de los recursos humanos y económicos, especialmente en los pacientes y en las cirugías más complejas.
A consecuencia del proceso epidemiológico de transición demográfica que ha vivido nuestro país, se estima que cerca de un tercio de los pacientes sometidos a una cirugía son adultos mayores, quienes en un 50\% requerirán una cirugía antes de morir[7]. Esto conlleva a que la complejidad en la atención de salud y el nivel de recursos utilizados en pabellón es significativamente mayor en comparación a 10 o 20 años atrás[2],[3],[7]. Los adultos mayores presentan factores que aumentan la morbilidad y mortalidad perioperatoria en comparación a la población más joven, debido a la menor reserva fisiológica y acondicionamiento físico relacionada con la edad avanzada, que puede ser agravada por las comorbilidades, el deterioro cognitivo, la fragilidad, la polifarmacia, entre otros[3]. Además, estos pacientes frecuentemente se someten a procedimientos quirúrgicos complejos y/o de urgencia[8]. Lamentablemente, en nuestro país no existen estudios que analicen la mortalidad postoperatoria en adultos mayores y sus factores asociados. Por ello, es relevante comenzar a reportar dichos análisis con la finalidad de evaluar el resultado de nuestra acción quirúrgica y enfocar adecuadamente los recursos utilizados en este subgrupo de pacientes, que a futuro será el prioritario[9].

En el Hospital Clínico de la Universidad de Chile 
(HCUCH) se realizan cerca de 16.000 cirugías al año y aproximadamente un tercio de estas son en pacientes adultos mayores (fuente: estadística de pabellones quirúrgicos). Dado que es una población significativa, se diseñó un estudio con el objetivo de determinar la tasa de mortalidad al primer año postoperatorio en una muestra aleatoria de pacientes mayores de 65 años sometidos a una cirugía bajo una anestesia general o regional.

\section{Material y Métodos}

Tras la aprobación del comité de ética del HCUCH (número OAIC 922/2017), se procedió a la realización de un estudio observacional analítico de una cohorte histórica entre el período de agosto de 2015 y julio de 2016. Se obtuvo la información de todos los pacientes mayores de 65 años sometidos a alguna cirugía que requirió de anestesia general y/o regional durante dicho periodo en nuestro centro y se incluyeron tanto procedimientos electivos como de urgencia. En total se identificaron 2.832 pacientes que cumplieron con los criterios antes mencionados pacientes, la que fue finalmente analizada. Por los recursos limitados, se tomó la decisión de analizar una muestra de la población. A partir de los 2.832 pacientes mencionados se obtuvo de manera aleatoria simple una muestra de 235 pacientes. Esta estrategia de muestreo ha sido discutida en la literatura[10]. Con el objetivo de evitar sesgos estacionales a lo largo del año, la muestra fue obtenida a partir de la población que se somete a cirugía durante un año completo.

Se revisaron las fichas clínicas electrónicas de todos los pacientes seleccionados en la plataforma de Ticares (ficha electrónica del HCUCH). Se registró para cada paciente, la edad al momento de la cirugía, antecedentes mórbidos, diagnóstico actual, complejidad de la cirugía[11] y anestesia, y duración de la intervención. Los antecedentes mórbidos de cada paciente de la muestra se consignaron para calcular el Charlson Comorbidity Index (CCI)[12], con la finalidad de estimar el riesgo de mortalidad de los pacientes en base a sus comorbilidades[13]. Este índice consiste en la ponderación de 19 condiciones médicas: edad, diabetes mellitus, enfermedad hepática, tumor sólido, SIDA $(\mathrm{VIH})$, enfermedad renal crónica moderada a severa, insuficiencia cardiaca congestiva, IAM, enfermedad pulmonar obstructiva crónica, enfermedad vascular periférica, accidente cerebrovascular o ataque isquémico transitorio, demencia, hemiplejia, enfermedad del tejido conectivo, leucemia, linfoma maligno y úlcera péptica. Finalmente, se consignó la mortalidad mediante la información del Registro Civil de Chile. Además, como objetivos secundarios, se describió la causa de muerte a partir de la información consignada en la ficha clínica electrónica y del certificado de defunción; se caracterizaron las variables clínicas y demográficas de los pacientes fallecidos y se compararon con los pacientes que no fallecieron.

El desenlace primario fue mortalidad al año tras la cirugía. Dado que la mortalidad reportada es aproximadamente $10 \%$ en adultos mayores de 65 años[4], entonces se calculó una muestra de 235 pacientes para detectar dicha tasa de mortalidad con un margen de $\pm 4 \%$, considerando una pérdida del $10 \%$, un error alfa de 0,05 y un poder del 80\%. Este cálculo se realizó con la plataforma Clinical.com/Stats/Samplesiz.aspx. Los datos continuos fueron expresados mediante promedios y desviación estándar. Los datos discontinuos se expresaron con medianas, mínimos y máximos. Finalmente, las variables categóricas se describieron con porcentaje. Para los análisis se utilizó el software Graphpad Prism 8.0.

\section{Resultados}

Se estudiaron finalmente un total de 233 pacientes, dado que 2 sujetos se excluyeron porque su cirugía no fue realizada con anestesia general y/o regional. El grupo de pacientes se caracterizó por tener un promedio de edad de 73,1 \pm 6,3 años, 52,4\% era de sexo femenino, una mediana de puntaje $\mathrm{CCl}$ de 4 (2-11) puntos, 24\% fue sometido a una cirugía de alta complejidad, 65,7\% fue intervenido quirúrgicamente bajo anestesia general y $34,3 \%$ bajo anestesia regional, con una mediana de tiempo operatorio de 74 (10-460) minutos y una mediana de días de hospitalización y posoperatorio de 3 (0-127) y 2,2 (0-52), respectivamente.

En total fallecieron $14(6,0 \%)$ pacientes tras 1 año desde el momento de la cirugía, mientras que sólo $3(1,3 \%)$ sujetos fallecieron durante los primeros 30 días postoperatorios. Los pacientes que fallecieron en el primer año posoperatorio tienden a ser más añosos y a tener un mayor $\mathrm{CCl}$ comparado con quienes no fallecieron $(79,1 \pm 9,9$ años versus $72,7 \pm 5,8$ años; $\mathrm{CCl}$ de 7,5 (3-10) puntos versus 4,0 (2-11) puntos). Específicamente, entre los pacientes que fallecieron, se encontró $21 \%$ con diagnóstico preoperatorio de demencia comparado con $1 \%$ en el grupo de pacientes no fallecidos, así como también, $29 \%$ de los pacientes que fallecieron tenían un tumor sólido con metástasis versus $2 \%$ en los pacientes que no fallecieron (Tabla 1). Sin embargo, a partir del análisis des- 


\begin{tabular}{lcc}
\multicolumn{2}{l}{$\begin{array}{c}\text { Tabla 1. Características evaluadas en el Charlson Comorbidity Index entre los pacientes que fallecieron al año } \\
\text { tras la cirugía y los que no fallecieron }\end{array}$} & $\begin{array}{c}\text { Vivos } \\
\text { Característica }\end{array}$ \\
DM tipo 2 & $4 / 14(29 \%)$ & $46 / 218(21 \%)$ \\
IAM & $2 / 14(14 \%)$ & $9 / 217(4 \%)$ \\
Insuficiencia cardiaca & $0 / 14(0 \%)$ & $9 / 216(4 \%)$ \\
Enfermedad arterial oclusiva & $1 / 14(7 \%)$ & $8 / 217(4 \%)$ \\
AVE o AIT & $1 / 14(7 \%)$ & $12 / 217(6 \%)$ \\
Demencia & $3 / 14(21 \%)$ & $3 / 218(1 \%)$ \\
EPOC & $0 / 14(0 \%)$ & $7 / 210(3 \%)$ \\
Enfermedad del tejido conectivo & $0 / 14(0 \%)$ & $2 / 218(1 \%)$ \\
Úlcera péptica & $0 / 14(0 \%)$ & $2 / 218(1 \%)$ \\
Enfermedad hepática & $1 / 14(7 \%)$ & $6 / 218(3 \%)$ \\
Hemiplejia & $1 / 14(7 \%)$ & $5 / 218(2 \%)$ \\
IRC moderada a severa & $1 / 14(7 \%)$ & $9 / 218(4 \%)$ \\
Tumor sólido localizado & $6 / 14(43 \%)$ & $43 / 218(20 \%)$ \\
Tumor sólido con metástasis & $4 / 14(29 \%)$ & $4 / 218(2 \%)$ \\
Leucemia & $0 / 14(0 \%)$ & $1 / 218(0 \%)$ \\
Linfoma & $0 / 14(0 \%)$ & $3 / 218(1 \%)$ \\
SIDA & $0 / 14(0 \%)$ & $0 / 217(0 \%)$ \\
\hline
\end{tabular}

DM: diabetes mellitus; IAM: infarto agudo al miocardio; AVE: accidente cerebrovascular; AIT: accidente isquémico transitorio; EPOC: enfermedad pulmonar obstructiva crónica; IRC: insuficiencia renal crónica; SIDA: síndrome de inmunodeficiencia adquirida.

criptivo no impresiona haber una diferencia entre ambos grupos en cuanto a la complejidad de las cirugías $(21,4 \%$ y $28,8 \%$ fueron sometidos a cirugías de alta complejidad en el grupo de fallecidos y no fallecidos, respectivamente). Lo mismo en cuanto a la duración de las cirugías (fallecidos: 67 (15-292) min versus no fallecidos 74 (10-460) min).

Los pacientes que fallecieron durante el primer año posoperatorio se sometieron a 3 cirugías de alta complejidad y a 11 cirugías de baja y mediana complejidad (Tabla 2). Las cirugías de alta complejidad fueron: una colectomía parcial derecha, una fijación de columna lumbar y una nefrectomía radical ampliada. Las otras cirugías fueron: tres procedimientos bajo anestesia general de colangiopancreatografía retrógrada endoscópica (CPRE), un aseo quirúrgico de un pie diabético, un retiro de prótesis vascular periférica, una gastrostomía, una pleurodesis, una cistoscopía, una instalación de prótesis para una fístula arterio-venosa, una artroscopía de hombro y un drenaje de hematoma subdural. Al analizar las causas de muerte, se evidencia que, en los tres pacien- tes sometidos a cirugías de alta complejidad, uno falleció por una complicación quirúrgica infecciosa 95 días tras la cirugía, otro falleció por un cuadro de neumonía 196 días tras la intervención quirúrgica y el último paciente falleció por la progresión de su cáncer de colon a los 235 días posoperatorios. En cuanto a los otros 11 pacientes, tres de ellos fallecieron en los primeros 30 días posoperatorio dado que las cirugías fueron de salvatajes en cuadros graves: drenaje de un hematoma subdural severo en un paciente anticoagulado de 96 años; cistoscopía por una hematuria macroscópica en un paciente séptico, anticoagulado y con un accidente cerebrovascular agudo; y gastrostomía en un paciente con falla orgánica múltiple que fue dado de alta, mientras otros cuatro fallecieron por la progresión de su cáncer (tres CPRE y pleurodesis) entre los días 182 y 258 posteriores a la intervención. Finalmente, los cuatro pacientes restantes fallecieron a los 97 días del postoperatorio por un probable IAM, a los 266 días por insuficiencia respiratoria y a los 336 días por un IAM, y una infección de la prótesis vascular. 
Tabla 2. Caracterización de los pacientes fallecidos en cuanto a tipos de cirugías, diagnóstico, día posoperatorio de muerte y causa de muerte

\begin{tabular}{|c|c|c|c|}
\hline Cirugía & Diagnóstico & Día & $\begin{array}{l}\text { Causa de muerta } \\
\text { (Registro Civil) }\end{array}$ \\
\hline Cistoscopía & Hematuria macroscópica & 3 & $\begin{array}{l}\text { Accidente cerebrovascular isqué- } \\
\text { mico más sepsis severa por pros- } \\
\text { tatitis }\end{array}$ \\
\hline Drenaje de hematoma subdural & Hematoma subdural crónico & 6 & $\begin{array}{l}\text { Hematoma subdural crónico rea- } \\
\text { gudizado con hemorragia suba- } \\
\text { racnoidea }\end{array}$ \\
\hline Gastrostomía & Trastorno deglución & 26 & $\begin{array}{l}\text { Deficiencia orgánica múltiple/de- } \\
\text { terioro cognitivo }\end{array}$ \\
\hline Fijación de columna lumbar & $\begin{array}{l}\text { Espondilolistesis L4-L5 con este- } \\
\text { nosis asociada }\end{array}$ & 95 & $\begin{array}{l}\text { Infección herida operatoria con } \\
\text { shock séptico }\end{array}$ \\
\hline Retiro prótesis con aseo quirúrgico & $\begin{array}{l}\text { Pie diabético con infección de } \\
\text { prótesis antigua }\end{array}$ & 97 & $\begin{array}{l}\text { Paro cardiorrespiratorio / Insufi- } \\
\text { ciencia cardiaca }\end{array}$ \\
\hline Colangiopancreatografía retrógrada & $\begin{array}{l}\text { Colangiocarcinoma con ictericia } \\
\text { obstructiva }\end{array}$ & 182 & Cáncer de vesícula biliar etapa IV \\
\hline $\begin{array}{l}\text { Nefrectomía radical ampliada más } \\
\text { segmentectomía hepática }\end{array}$ & Liposarcoma retroperitoneal & 196 & $\begin{array}{l}\text { Sepsis foco respiratorio/neumo- } \\
\text { nía/hemorragia digestiva baja }\end{array}$ \\
\hline $\begin{array}{l}\text { Pleurodesis por video toracotomía } \\
\text { con biopsia pleural }\end{array}$ & Derrame pleural & 198 & Cáncer de mama \\
\hline Colectomía parcial derecha & Cáncer de colon & 235 & Cáncer de colon \\
\hline $\begin{array}{l}\text { Colangiopancreatografía retrógrada } \\
\text { con instalación de stent }\end{array}$ & $\begin{array}{l}\text { Cáncer de páncreas con metásta- } \\
\text { sis hepática }\end{array}$ & 249 & Cáncer de páncreas etapa IV \\
\hline Artroplastia de hombro & $\begin{array}{l}\text { Fractura de la epífisis superior del } \\
\text { húmero }\end{array}$ & 249 & IAM \\
\hline $\begin{array}{l}\text { Colangiopancreatografía retrógrada } \\
\text { con instalación de stent }\end{array}$ & $\begin{array}{l}\text { Colangitis por colangiocarcino- } \\
\text { ma }\end{array}$ & 258 & Colangiocarcinoma \\
\hline Aseo quirúrgico & $\begin{array}{l}\text { Infección de herida operatoria } \\
\text { posamputación de ortejo de EEll }\end{array}$ & 266 & $\begin{array}{l}\text { Paro cardiorrespiratorio/insufi- } \\
\text { ciencia respiratoria crónica }\end{array}$ \\
\hline Instalación de prótesis vascular & Complicación catéter diálisis & 336 & $\begin{array}{l}\text { Infección prótesis hemodiálisis/ } \\
\text { shock séptico }\end{array}$ \\
\hline
\end{tabular}

\section{Discusión}

A partir de los resultados obtenidos se concluye que la mortalidad postoperatoria está por debajo de lo reportado y, como era de esperar, aumenta con la edad y las comorbilidades de los pacientes. Por otro lado, en nuestro estudio, no se encontró una asociación entre mortalidad al año tras la cirugía con la duración ni con la complejidad del procedimiento quirúrgico. Finalmente, es destacable el hecho que ningún paciente falleció en el intraoperatorio, lo que nuevamente se corresponde con lo reportado.

La mortalidad postoperatoria al año de nuestra muestra fue de $6 \%$, lo cual es menor a lo reportado previamente en una población similar a la nues- tra. Sin embargo, este dato debe ser tomado con cautela dada la naturaleza metodológica de nuestro estudio. Dado que el tamaño muestral fue calculado considerando una dispersión de un $\pm 4 \%$, nuestros resultados estarían dentro del margen de precisión de la muestra. Ahora bien, nuestra tasa de mortalidad se encuentra de todas formas en el límite inferior de lo reportado. Esto se puede explicar por diversos factores. Una posible causa puede ser la menor fragilidad de la población atendida en el $\mathrm{HCUCH}$ en comparación a otros centros hospitalarios, dado que la población de nuestro centro tiene un mayor nivel de escolaridad y un mayor nivel socioeconómico que los pacientes atendidos en el sistema público de salud[14]. Otro factor que puede haber influido en el re- 
sultado obtenido es el hecho que en nuestro centro el porcentaje de cirugías de urgencia es bajo, como las cirugías de politraumatismos y las cirugías cardiacas, las cuales se han descrito que aumentan la mortalidad perioperatoria[4],[15],[16].

En cuanto a las causas de mortalidad es interesante destacar un estudio de cohorte prospectivo realizado en el Hospital Universitario Virgen del Rocío de Sevilla[17], el cual consignó que el 9\% de los pacientes, con criterios de sepsis en su entrada a la $\mathrm{UCl}$, fallecieron en el seguimiento de 2 años tras el alta hospitalaria, aumentando hasta el $20 \%$ en los mayores de 65 años. Los predictores independientes de mortalidad a 2 años en los pacientes ancianos fueron: insuficiencia cardiaca crónica, insuficiencia renal aguda, insuficiencia respiratoria aguda y antibioterapia empírica inadecuada, asociando esta última con una reducción del doble en la supervivencia a los 2 años[17]. En nuestra muestra, los pacientes que fallecieron al año tras la cirugía tuvieron un CCI mayor que los pacientes que no fallecieron. Las diferencias especialmente se debieron a que los pacientes que fallecieron tenían en mayor proporción un tumor sólido con metástasis y demencia. Por lo tanto, efectivamente los pacientes que fallecieron tenían un nivel de comorbilidad mayor en comparación que aquellos que no fallecieron, aunque dada la baja proporción de fallecidos no fue posible realizar análisis estadístico para comparar ambos grupos.

En cuanto a las causas específicas de muerte, destaca que la principal fue la progresión de la enfermedad oncológica de base (4 pacientes de 14 fallecidos), mientras otros 5 pacientes fallecieron tras los primeros 30 días postoperatorios, por causas de muertes comunes en los adultos mayores, como son las neumonías y el IAM. Por otro lado, otros 3 pacientes fallecieron por causas derivadas a su condición de gravedad por un shock séptico o hemorrágico, en la que la cirugía fue una medida terapéutica inefectiva. Finalmente, una paciente falleció directamente de una complicación infecciosa de la cirugía. En suma, en nuestra muestra los pacientes fallecieron principalmente por la patología de base que justificó la cirugía, más que una complicación posquirúrgica. Esta observación no se condice con lo reportado en cuanto a que las principales causas de muerte postoperatorias, especialmente en los primeros 30 días tras la cirugía, son las complicaciones miocárdicas, tales como IAM y paro cardiorrespiratorio de causa cardiaca[1],[18]. Esta diferencia puede ser secundario a que nuestra muestra no tuvo un poder suficiente para detectar como causa de mortalidad a las complicaciones postoperatorias cardiacas. Pero es de resaltar que la principal causa de muerte observada fue la progresión de la enfermedad como un cáncer metastizado, lo que sí ha sido reportado previamente[1]. Sin duda alguna, la utilización de bases electrónicas en cada uno de los centros permite realizar estudios con un tamaño muestral mayor, lo que es absolutamente necesario para enfocar nuestro manejo perioperatorio en relación con las causas de morbimortalidad de nuestra población.

Finalmente, es sabido que la mortalidad intraoperatoria actualmente es muy poco frecuente, debido a que los incidentes intraoperatorios son habitualmente controlados[18]. Acorde con esto, en nuestro estudio ningún paciente falleció en el intraoperatorio. Sin embargo, se han descrito eventos durante la cirugía como factores que predicen la mortalidad postoperatoria a largo plazo, tales como episodios de hipotensión[19]-[21], aumento en el tiempo de mayor profundidad anestésica[4],[22],[23]. El análisis de dichos eventos, entre otros, es material de estudio para futuras investigaciones, con el objetivo de conocer más factores asociados a la mortalidad postoperatoria en pacientes de 65 años y más, para enfocar posibles intervenciones. Pero sin duda alguna, la progresión de la enfermedad de base, aparentemente, es lo que determina en mayor medida el desenlace de los pacientes.

Nuestro estudio tiene varias limitaciones, las cuales derivan principalmente del diseño retrospectivo a partir de una muestra pequeña aleatoria de una cohorte cerrada. Esto lleva a la existencia de potenciales confundentes como el tipo de cirugía que se realiza en un centro en particular, el tipo de hospitalización (no se incluyen pacientes ambulatorios) y los riesgos basales de los pacientes, entre otros. Estos factores pueden llevar a sesgos en la interpretación de los resultados y, por ende, deben ser tomados con cautela. Ahora bien, dada la inexistencia de datos nacionales al respecto, este trabajo es una primera aproximación a nuestra realidad, cuya finalidad es generar hipótesis para estudios poblacionales mayores a nivel nacional.

En conclusión, en nuestro estudio se observó una mortalidad postoperatoria al año menor a lo reportado en pacientes adultos mayores. La mortalidad fue mayor en pacientes de mayor edad y con mayores comorbilidades, especialmente con tumores sólidos con metástasis. Además, las causas de muerte encontradas en nuestro estudio fueron principalmente secundarias a la progresión de la enfermedad de base o por patologías propias de los adultos mayores, más que complicaciones perioperatorias. La realización de estudios de morbimortalidad perioperatoria en cada uno de nuestros centros permitirá enfocar nuestros manejos en relación con las causas relevantes de nuestra población. 


\section{Referencias}

1. Sessler DI. The 2016 John W. Severinghaus lecture on translational research. Anesthesiology. 2017 Jun;126(6):995-1004. https://doi.org/10.1097/ ALN.0000000000001603 PMID:28358749

2. Weiser TG, Semel ME, Simon AE, Lipsitz SR, Haynes AB, Funk LM, et al. In-hospital death following inpatient surgical procedures in the United States, 1996-2006. World J Surg. 2011 Sep;35(9):1950-6. https://doi. org/10.1007/s00268-011-11695 PMID:21732207

3. Jerath A, Austin PC, Wijeysundera DN. Days Alive and Out of Hospital: Validation of a Patient-centered Outcome for Perioperative Medicine. Anesthesiology. 2019 Jul;131(1):84-93. https://doi.org/10.1097/ ALN.0000000000002701 PMID:31094760

4. Monk TG, Saini V, Weldon BC, Sigl JC. Anesthetic management and one-year mortality after noncardiac surgery. Anesth Analg. 2005 Jan;100(1):4-10. https://doi.org/10.1213/01. ANE.0000147519.82841.5E PMID:15616043

5. Noordzij PG, Poldermans D, Schouten O, Bax JJ, Schreiner FA, Boersma E. Postoperative mortality in The Netherlands: a population-based analysis of surgery-specific risk in adults. Anesthesiology. 2010 May;112(5):1105-15. https://doi.org/10.1097/ ALN.0b013e3181d5f95c PMID:20418691

6. Devereaux PJ, Biccard BM, Sigamani A, Xavier D, Chan MT, Srinathan SK, et al.; Writing Committee for the VISION Study Investigators. Association of postoperative high-sensitivity troponin levels with myocardial injury and 30-day mortality among patients undergoing noncardiac surgery. JAMA JAMA. 2017 Apr;317(16):164251. https://doi.org/10.1001/ jama.2017.4360 PMID:28444280

7. Castellanos-Olivares A, SánchezRuíz JG, Gómez-Sánchez G, Salgado-Figueroa M. Prevalencia de Comorbilidades En El Paciente Geriátrico y Pronóstico Postoperatorio. Vol 40. Medigraphic; 2017. Accessed September 11, 2020. www.medigraphic.org.mx

8. Glance LG, Lustik SJ, Hannan EL, Osler TM, Mukamel DB, Qian F, et al. The Surgical Mortality Probability Model: derivation and validation of a simple risk prediction rule for noncardiac surgery. Ann Surg. 2012 Apr;255(4):696702. https://doi.org/10.1097/ SLA.0b013e31824b45af PMID:22418007

9. Medina-Franco $\mathrm{H}$, Ramos-De la Medina A, Torres-Villalobos G, Tapia-Cid de León H. Factores asociados a mortalidad en cirugía mayor: Análisis retrospectivo en un centro de referencia. Rev Investig Clin. 2006;58(1):9-14. Accessed September 11, 2020. http://www.scielo.org.mx/scielo.php?script=sci_arttext\&pid =S0034-83762006000100002

10. Banerjee A, Chaudhury S. Statistics without tears: populations and samples. Ind Psychiatry J. 2010 Jan;19(1):60-5. https://doi. org/10.4103/0972-6748.77642 PMID:21694795

11. Smilowitz NR, Berger JS. Perioperative Cardiovascular Risk Assessment and Management for Noncardiac Surgery: A Review. JAMA. 2020 Jul;324(3):279-90. https://doi. org/10.1001/jama.2020.7840 PMID:32692391

12. Charlson ME, Pompei $P$, Ales KL, MacKenzie CR. A new method of classifying prognostic comor- bidity in longitudinal studies: development and validation. J Chronic Dis. 1987;40(5):373-83. https://doi.org/10.1016/00219681(87)90171-8 PMID:3558716

13. Turrentine FE, Wang $H$, Simpson VB, Jones RS. Surgical risk factors, morbidity, and mortality in elderly patients. J Am Coll Surg. 2006 Dec;203(6):865-77. https://doi.org/10.1016/j. jamcollsurg.2006.08.026 PMID:17116555

14. Tapia CP, Valdivia-Rojas $Y$, Varela HV, Carmona AG, Iturra VM, Jorquera MC. [Rates of frailty among older people ascribed to Chilean primary care clinics]. Rev Med Chil. 2015 Apr;143(4):45966. https://doi.org/10.4067/ S0034-98872015000400007 PMID:26204537

15. Pearse RM, Moreno RP, Bauer $P$, Pelosi P, Metnitz P, Spies $C$, et al.; European Surgical Outcomes Study (EuSOS) group for the Trials groups of the European Society of Intensive Care Medicine and the European Society of Anaesthesiology. Mortality after surgery in Europe: a 7 day cohort study. Lancet. 2012 Sep;380(9847):1059-65. https://doi.org/10.1016/ S0140-6736(12)61148-9 PMID:22998715

16. Siregar S, Groenwold RH, de Mol BA, Speekenbrink RG, Versteegh MI, Brandon Bravo Bruinsma GJ, et al. Evaluation of cardiac surgery mortality rates: 30-day mortality or longer follow-up? Eur J Cardiothorac Surg. 2013 Nov;44(5):875-83. https:// doi.org/10.1093/ejcts/ezt119 PMID:23471150

17. Carbajal-Guerrero J, Cayuela-Domínguez A, Fernández-García E, Aldabó-Pallás T, Márquez-Vácaro JA, Ortiz-Leyba C, et al. Epidemiología y pronóstico tardío de la sepsis en ancianos. Med Inten- 
siva. 2014 Jan-Feb;38(1):21-32. https://doi.org/10.1016/j. medin.2012.12.006 PMID:23462427

18. Devereaux PJ, Sessler DI. Cardiac complications in patients undergoing major noncardiac surgery. Longo DL, ed. N Engl J Med. 2015;373(23):2258-2269. https://doi.org/10.1056/NEJMra1502824.

19. Monk TG, Bronsert MR, Henderson WG, Mangione MP, Sum-Ping ST, Bentt DR, et al. Association between intraoperative hypotension and hypertension and 30-day postoperative mortality in noncardiac surgery. Anesthesiology. 2015 Aug;123(2):307-19. https://doi.org/10.1097/ ALN.0000000000000756 PMID:26083768
20. Bijker JB, van Klei WA, Vergouwe $Y$, Eleveld DJ, van Wolfswinkel L, Moons KG, et al. Intraoperative hypotension and 1-year mortality after noncardiac surgery. Anesthesiology. 2009 Dec;111(6):1217-26. https://doi.org/10.1097/ ALN.0b013e3181c14930 PMID:19934864

21. Walsh M, Devereaux PJ, Garg AX, Kurz A, Turan A, Rodseth $\mathrm{RN}$, et al. Relationship between intraoperative mean arterial pressure and clinical outcomes after noncardiac surgery: toward an empirical definition of hypotension. Anesthesiology. 2013 Sep;119(3):507-15. https://doi.org/10.1097/ ALN.0b013e3182a10e26 PMID:23835589

22. Lindholm ML, Träff S, Granath F,
Greenwald SD, Ekbom A, Lennmarken $C$, et al. Mortality within 2 years after surgery in relation to low intraoperative bispectral index values and preexisting malignant disease. Anesth Analg. 2009 Feb;108(2):508-12. https://doi.org/10.1213/ ane.0b013e31818f603c PMID:19151279

23. Kertai MD, Pal N, Palanca BJ, Lin N, Searleman SA, Zhang L, et al.; B-Unaware Study Group. Association of perioperative risk factors and cumulative duration of low bispectral index with intermediate-term mortality after cardiac surgery in the BUnaware Trial. Anesthesiology. 2010 May;112(5):1116-27. https://doi.org/10.1097/ ALN.0b013e3181d5e0a3 PMID:20418692 